\title{
Fluorescent in situ hybridization of mitochondrial DNA and RNA
}

\author{
Lukáš Alán, Jaroslav Zelenka, Jan Ježek, Andrea Dlasková and Petr Ježek \\ Department No. 75, Institute of Physiology, Academy of Sciences of the Czech Republic, Prague, Czech Republic
}

To reveal nucleic acid localization in mitochondria, we designed molecular beacon fluorescent probes against: i) the light strand complementary to ND5 mitochondrial DNA (mtDNA) gene (annealing also to corresponding mRNA); ii) displacement (D) loop 7S DNA (annealing also to parallel heavy strand mtDNA and corresponding light strand transcript); iii) the proximal D-loop heavy strand displaced by the light strand promoter minor RNA. Confocal microscopy demonstrated ND5 probe spreading (less for other probes) in mitochondrial reticulum tubules but upon RNase A treatment all probes contoured mtDNA nucleoid localization. DNase I spread the signal over mitochondrial tubules. Future applications are discussed.

Keywords: mitochondrial DNA and RNA, nucleoids of mitochondrial DNA, molecular beacon fluorescent hybridization probes, confocal microscopy, transcription and replication intermediates

Received: 30 August, 2010; revised: 04 November, 2010; accepted: 25 November, 2010; available on-line: 29 November, 2010

\section{INTRODUCTION}

Mitochondrial DNA (mtDNA) most probably determines aging to some extent and hence the lifespan of living organisms (Holt, 2010; Tuppen et al., 2010). In turn, mtDNA genetics is characterized by robustness due to multiple mtDNA copies (typically 1000 to 10000 ) existing in a single cell (Holt et al., 2007; Holt, 2010; Tuppen et al., 2010) and due to a purifying bottleneck effect during embryonic and fetal development (Cree et al. 2008; Stewart et al., 2008). Moreover, mtDNA mutations have been implicated in mitopathies and other diseases, namely those originating from oxidative stress - a constant inevitable superoxide production is a byproduct of respiratory chain function and "tax for life“ (Lu et al., 2000; Zhadanov et al., 2007; Scaglia \& Wong, 2008; Holt, 2010; Tuppen et al., 2010). It is surprising how little information has been gathered concerning mitochondrial genetics, about details of transcription and replication mechanisms of mtDNA (Fig. 1A, B), and the structure of mtDNA organized in so-called mt nucleoids (Holt et al. 2007; Bogenhagen et al., 2008).

An originally proposed asynchronous strand displacement replication model for $\mathrm{mtDNA}$ was later questioned for the lacking existence of its single strand asynchronous intermediates (Yasukawa et al., 2006; Hyvärinen et al., 2007, Holt, 2009). This model assumed that DNA synthesis is primed by transcription through the $\mathrm{H}$ strand origin (OriH) within the displacement (D) loop (Shadel \& Clayton, 1997; Fig. 1B). Unidirectional synthesis is supposed to proceed along the template while displacing the opposite strand. After two-thirds around the 16-kb circular human mt genome, the nascent $\mathrm{H}$ strand should be replicated and the L strand origin OriL should be exposed, allowing initiation of the nascent $\mathrm{L}$ strand synthesis. Elongation of the second strand then should continue along the previously displaced single-stranded (ss) template in the opposite direction. The leading strand and lagging strand synthesis are supposed to be independent in mitochondria. Both strands should be extended in an asymmetric manner until two daughter molecules are formed (Clayton, 2003; Falkenberg et al., 2007). Mitochondrial RNA polymerase serves as a primase required for DNA synthesis at OriL (Fusté et al., 2010).

Mitochondria contain the apparatus for Okazaki fragment maturation, although it is not clear whether this mechanism is in effect (Holt, 2009). Here, a transcript from the L strand promoter (LSP) serves as a primer for $\mathrm{H}$ strand replication while forming the RNA/DNA hybrid prior to OriH, and hence forming the D-loop (Fig. 1A, B). The existence of two promoters, LSP and $\mathrm{LSP}_{\text {minor }}$ (Chang et al., 1986), has been suggested to produce both a polycistronic transcript (LSP RNA) and replication primer. According to a processing model assuming self-cleavage of polycistronic RNA based on self-splicing sequences including those in tRNA genes (Chang \& Clayton, 1985), one transcriptional event gives rise to the priming RNA plus the precursor RNA for structural genes (ND6 in the case of LSP RNA, remaining on HSP RNA). Transcription from the LSP ${ }_{\text {maior }}$ promoter, when it is self-cleaved from the transcript, could produce priming RNA preceding 7S DNA (Fig. 1A). Thus the priming RNA coincides with the supposed 5' end of starting $\mathrm{H}$ strand replication and, consequently, it should compete with the unprocessed (uncleaved polycistronic) transcript and regulate replication of OriH. In the case of $\mathrm{LSP}_{\text {minor }}$, no such regulation has been observed and probably the corresponding RNA serves as a primer in all occasions (Chang \& Clayton, 1985).

One of the alternatives, the strand-coupled bidirectional replication model (Bowmaker et al., 2003; Brown et al., 2005; 2008; Brown \& Clayton, 2006; Yasukawa et al., 2006; Pohjoismäki et al., 2010) assumes that bidirectional replication is initiated from a zone near OriH followed by the progression of the two forks around the mtDNA circle (Bowmaker et al., 2003). This model proposes that

\footnotetext{
$\star$ e-mail: jezek@biomed.cas.cz
$\star$ This work was presented in the poster form at the 16th European Bioenergetics Conference (Warsaw, 2010); abstract in Biochim Biophys Acta, 1797 (Suppl): 105 (2010).

Abbreviations: D-loop, displacement loop; ds, double-stranded; EGFP, enhanced green fluorescence protein; FISH, fluorescence in situ hybridization; HSP, H strand promoter; LSP, L strand promoter; $\mathrm{mt}$, mitochondrial; mtDNA, mitochondrial DNA; OriH, $\mathrm{H}$ strand origin; OriL, L strand origin; PBS, phosphate buffered-saline; RITOLS, RNA incorporations throughout of lagging strand; ss, singlestranded; TFAM, transcription factor-A, mitochondrial.
} 
L strand replication initiation can occur at various locations other than OriL, as alternative L strand origins (altOriL; Brown \& Clayton, 2006; Holt et al., 2007; Holt, 2009; Fig. 1B). These altOriL modifications require, on occasion, a reduction in the asymmetry and a mechanism to initiate $\mathrm{L}$ strand replication at locations other than OriL. This model is supported by the revealed two classes of double-stranded (ds) replication intermediates, the first class consisting of the predicted products of conventional strand-coupled replication (remaining after RNA loss during isolation and processing; Holt et al., 2000; Kajander et al., 2001; Bowmaker et al., 2003; Reyes et al., 2005); and the second, extended RNA segments encompassing the entire lagging strand, i.e., RNA incorporations throughout of lagging strand (RITOLS; Yang et al., 2002; Bowmaker et al., 2003). The mechanism by which the RNA lagging strand is created is unknown. The nascent RNA strand merely protects the lagging strand template until the second-strand DNA synthesis is initiated. Other remnant RNA/DNA hybrid structures, called R-loops, have been identified to exist at a variety of locations throughout the $\mathrm{mt}$ genome due to retention of the RNA paired to its DNA template (Xu \& Clayton, 1996; Brown et al., 2008). The original role of R-loops should lie in priming the leading strand DNA synthesis. However, an RNase H-like activity exists which digests RNA only when it is hybridized to DNA. Hence, the putative RNase $\mathrm{H}$ probably generates short RNA primers when it comes in contact with the nascent RNA strands of mtDNA replicating via RITOLS. Consequently, potential primers are formed at dispersed sites. In conclusion, it is currently accepted that the conventional leading and lagging strand DNA synthesis may occur in parallel with RITOLS replication in mammalian mitochondria (Pohjoismäki et al., 2010).

The above described knowledge has been gathered mostly with isolated mtDNA and other entities such as isolated $\mathrm{mt}$ nucleoids. Visualization of, e.g., RNA/DNA hybrids in situ might contribute to confirming the gathered knowledge or obtaining more details. Also mt RNA existing in various forms, e.g., as polycistronic RNA (nascent at transcription) and as cleavage/self-cleavage products, i.e., tRNAs and mRNAs, have rarely been visualized and localized within the mitochondrial matrix. Therefore, we have initiated an effort in this path. Attempting to visualize mtDNA and certain forms of mitochondrial RNA, we designed three fluorescent probes for in situ hybridization (in fixed cells) based on the principle of molecular beacon (Santangelo et al., 2005). This is constructed so that complementary oligonucleotides flank the desired hybridizing sequence with the quencher located at the $3^{\prime}$ end and a desired fluorophore (Cy3 dye in our case) located at the 5 ' end of resulting constructs. The self-hybridization of the complementary oligonucleotides within the formed loop brings the quencher towards the fluorophore, so that the beacon probe does not emit fluorescence. Upon hybridization, the loop structure is aborted, hence the quencher does not quench the fluorescence of the $\mathrm{Cy} 3$ dye anymore. Note also that conventional confocal microscopy has a resolution of about $250 \mathrm{~nm}$, i.e., the space interval in which no two or more points could be distinguished from a single point. Consequently, no structure within a single $\mathrm{mt}$ nucleoid can be resolved. In spite of this, one still may use it in order to develop methodology before adapting these probes for super-resolution microscopic techniques. Here, we designed at first the "ND5 probe" to be annealed to the light (L, i.e., antisense) strand com- plementary to the ND5 mtDNA gene ( $\mathrm{H}$ strand $\mathrm{mtD}$ NA sequence), but also to RNAs containing the ND5 sequence. We clearly demonstrated that such a molecular beacon ND5 probe visualized objects co-localizing with tubules of mitochondrial reticulum (matrix space), thus reflecting HSP RNA and ND5 mRNA spreading localization in the matrix space. Two other probes, termed "7S DNA probe" and "proximal D-loop probe", designed against the $\mathrm{H}$ strand D-loop regions controlled by the light $(\mathrm{L})$ strand promoter major $\left(\mathrm{LSP}_{\text {major }}\right)$ and $\mathrm{LSP}_{\text {mi- }}$ nor, respectively, hybridize preferentially to mtDNA, since structures co-localizing with mitochondrial nucleoids were visualized by them. Nevertheless, LSP RNA, either within transcribing/replicating nucleoids or spread in the matrix space could also be visualized. In conclusion, we have laid out methodology required for future extension to super-resolution microscopy that might be able to resolve even distinct $\mathrm{mt}$ nucleoid types and structures such as RITOLS and R-loops on nucleoids of mtDNA.

\section{METHODS}

Cell model used. Human hepatocellular carcinoma HepG2 cells obtained from European culture collection (ECACC 85011430) were cultivated in DMEM without glucose supplemented with $3 \mathrm{mM}$ glutamine, $10 \%$ (v/v) glucose-free dialyzed fetal calf serum, $10 \mathrm{mM}$ Hepes, 10 $\mathrm{IU} / \mathrm{ml}$ penicillin, $100 \mu \mathrm{g} / \mathrm{ml}$ streptomycin and $10 \mathrm{mM}$ galactose, in order to force cells to oxidative phosphorylation (Rossignol et al., 2004). This treatment also activates mitochondrial biogenesis including mtDNA replication, hence it leads to more mt nucleoids.

Molecular beacon design and preparation. Revised Cambridge Reference Sequence of the human mtDNA (Andrews et al., 1999) was used and probes were designed against its regions as illustrated in Fig. 1A, B. The molecular beacon ND5 probe (No. 3 in Fig. 1) for annealing to the L strand (antisense) base pairs (bp) 12924 to 12948 complementary to a portion of the ND5 gene (H strand bp 12337 to 14148) was designed as follows (with complementary nucleotides in normal font and the ND5 sequence part in bold):

\section{5'-Cy3-CGA CGG AAG GGC TAT TTG TTG TGG GTC- TCG TCG-BHQ2-3'.}

This probe should also anneal to the mitochondrial polycistronic HSP RNA (Fig. 1A) which might be also a part of RITOLS (Fig. 1B) and the corresponding ND5 mRNA. The ND5 mRNA should not be localized only at $\mathrm{mt}$ nucleoids, but should be spread all over the matrix space and in $\mathrm{mt}$ ribosomes. In addition, replicating nucleoids are expected to give a more intensive signal, due to the doubling of the $\mathrm{L}$ strand.

Two other probes were designed for annealing to the D-loop region of $\mathrm{H}$ strand (bp 16024 to 574). Probe No. 2 (Fig. 1A), called "7S DNA probe", should anneal to the $\mathrm{H}$ strand bp 16109 to 16127 , which is the part of the nascent $7 \mathrm{~S}$ DNA displacing this $\mathrm{H}$ strand (Fig. 1A, B). Naturally, the probe should anneal also to the $7 \mathrm{~S}$ DNA. Moreover, the corresponding region of non-cod-

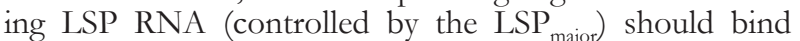
this probe as well, either before self-cleavage at the first tRNA gene location or afterwards (Fig. 1A). Therefore, the 7S DNA probe was designed as follows (with complementary pentanucleotides in normal font and the $7 \mathrm{~S}$ DNA sequence part in bold): 

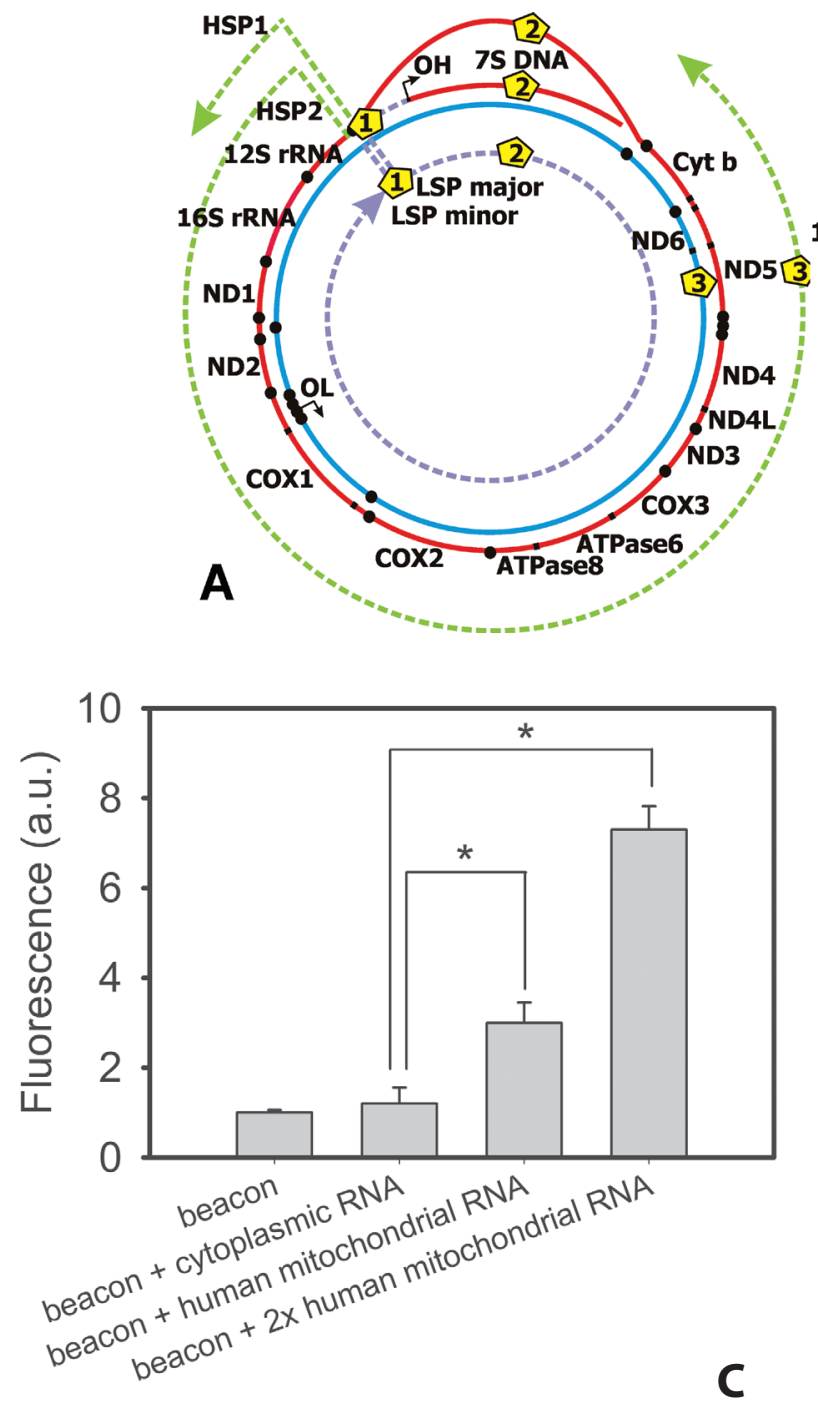

Figure 1. Scheme of possible hybridization of the developed probes to mitochondrial DNA and RNA upon transcription (A) and replication (B) and demonstration of hybridization specificity (C)

(A) Transcription schema illustrates the heavy $(\mathrm{H}$, red) and light ( $\mathrm{L}$, blue) strands of ds circular mtDNA, where borders between $\mathrm{mt}$ genes (their common abbreviations used in bold) are indicated by small black squares and tRNA genes as black dots; two possible polycistronic RNAs derived from them (HSP RNA, green, and LSP RNA, violet dashed lines) are illustrated with indicated transcription direction. The displacement (D) loop region contains permanent third strand with OriH $\left({ }_{1} \mathrm{OH}^{\prime \prime}\right)$. The possible hybridization positions of molecular beacon probes are denoted by numbers in yellow pentagons: 1) proximal D-loop probe, also termed $\mathrm{LSP}_{\text {minor }}$ RNA probe; 2) 7S DNA probe; and 3) ND5 probe. (B) Replication schema uses the same graphics as A). Here also RITOLS are illustrated by blue dashed line, while nascent $\mathrm{H}$ and $\mathrm{L}$ strand by red and blue dot-dashed line, respectively. For the strand-coupled bidirectional replication model, alternative origin sites ("Alt $\mathrm{OL}^{\prime \prime}$ ) besides the classic OriL ("OL") are depicted as the nascent $L$ strand starts to be synthesized at them and replaces protecting RNA, i.e., RITOLS. Theoretically, in replicating nucleoids the ND5 probe (No.3) could anneal to the corresponding RITOLS, to the nascent $L$ strand plus to the original $L$ strand. (C) Demonstration of hybridization specificity for the ND5 molecular beacon probe by comparing fluorescence of the ND5 probe mixed with RNA extract from mitochondria and cells depleted of mitochondria.

5'-Cy3-ACC GTA GCC ACC ATG AAT AT'T GTA CGGT-BHQ2-3'.

We expect that when, after cleavage, this non-coding LSP RNA diffuses out of $\mathrm{mt}$ nucleoids, its degradation might be faster than the degradation of mRNA. Faster degradation is also expected for the corresponding LSP $\mathrm{RNA}_{\text {minor }}$ located in the very proximal part of $\mathrm{D}$ loop. For this ultimate proximal part of D loop, we designed the third probe (probe No. 1, Fig. 1A) which should anneal to the $\mathrm{H}$ strand bp 524 to 547 . This is the portion of the D-loop initially displaced by the priming RNA portion of LSP RNA, to which this probe has to anneal as well (Fig. 1A). This "proximal D-loop probe" or "LSP ${ }_{\text {minor }}$ RNA probe" was designed as follows (with complementary pentanucleotides in normal font and the proximal D-loop sequence part in bold):

\section{5'-Cy3-ACC TGA CAC ACA CCG CTG CTA ACC CCA- 'TCA GGT-BHQ2-3'.}

Figure 1C demonstrates by fluorescence intensities that all three probes were fluorescent exclusively when hybridized to the sequences against which they were designed. In addition, in situ hybridization with a molecular beacon

probe designed against 28S rRNA (Alán et al., unpublished) and a random molecular beacon (Santangelo et al., 2005) gave no fluorescent signal in mitochondria.

Standardized mtFISH protocol. The standardized protocol for fluorescent in situ hybridization of $\mathrm{mtDNA}$ or mtRNA (mtFISH) was adjusted as follows: Cells were fixed in $0.3 \%$ glutaraldehyde in PBS for $20 \mathrm{~min}$ at $37^{\circ} \mathrm{C}$, followed by two washes with $1 \mathrm{mg} / \mathrm{ml} \mathrm{NaBH}_{4}$ in PBS for $15 \mathrm{~min}$ in order to reduce unbound glutaraldehyde. Afterwards, post-fixation nuclease treatment was performed in some experiments. Partial treatment with RNase A (400 $\mu \mathrm{g}$ per coverslip; Invitrogen), removing ssRNA was performed for two hours in PBS at $37^{\circ} \mathrm{C}$. To remove RNA from RNA/DNA hybrids we used digestion with RNase H (15 U per coverslip; Biolabs) for $30 \mathrm{~min}$ at $37^{\circ} \mathrm{C}$. Treatment with DNase I (200 U per coverslip; Top-Bio, Czech Republic), which digests dsDNA, proceeded for $30 \mathrm{~min}$ at $37^{\circ} \mathrm{C}$. The DNase I and RNase $\mathrm{H}$ treatments were performed in a reaction buffer prepared according to the manufacturer's protocol. Coverslips were pre-hybridized in $50 \%$ formamide with salmon sperm DNA and $0.2 \%$ Tween 20 in 2-fold concentrated saline sodium citrate $(2 \times \mathrm{SSC})$ buffer for $5 \mathrm{~min}$ at $72^{\circ} \mathrm{C}$. Molecular beacon (100 ng) in $50 \%$ formamide with $0.2 \%$ Tween in $2 \times \mathrm{SSC}$ buffer was added on the coverslip and hybridized overnight at $37^{\circ} \mathrm{C}$. Coverslips 
were then washed in $0.2 \%$ Tween with $50 \%$ formamide in $2 \times \mathrm{SSC}$ for $5 \mathrm{~min}$ at $42^{\circ} \mathrm{C}$, followed by three washes in $0.2 \%$ Tween in $2 \times \mathrm{SSC}$.

Confocal microscopy. An inverted confocal fluorescent Leica TCS SP2 AOBS microscope was employed with a PL APO $100 \times / 1.40-0.70$ oil immersion objective (a pinhole of 1 Airy unit). For Cy3 dye the excitation by a $1.2 \mathrm{~mW} \mathrm{He} / \mathrm{Ne}$ laser was set at $543 \mathrm{~nm}$. Matrixaddressed RoGFP, a modified EGFP of "type 1", a kind gift from Dr. Rodrigue Rossignol, Univ. Bordeaux 2 (France), was excited at $488 \mathrm{~nm}$ with a $20-\mathrm{mW}$ argon laser, to visualize tubules of mitochondrial reticulum (Rossignol et al., 2004). HepG2 cells pre-transfected with baculoviral particles (a kind gift of Dr. Werner Koopman, Nijmegen Centre for Molecular Life Sciences, Radboud University Nijmegen Medical Centre, Nijmegen, The Netherlands) coding for EGFP conjugated with mitochondrial transcription factor-A (TFAM) were used for visualization of $\mathrm{mt}$ nucleoids. Baculoviral particles were prepared on the basis of a modified Baculovirus vector containing a mammalian-active promoter (Koopman et al., 2007). Transfection was performed by incubating cells with baculoviral particles for $20 \mathrm{~h}$ to $24 \mathrm{~h}$. The culture medium contained $1.5 \mathrm{mM}$ sodium butyrate for proper expression of EGFP-TFAM. After transfection the cells were washed with PBS and fixed.

\section{RESULTS}

\section{Mitochondrial localization of hybridized molecular beacons}

Successful in situ hybridization is suggested by colocalization of the fluorescence of the ND5 molecular beacon with that of mitochondria addressed-RoGFP (Fig. 2A, B, C) and with EGFP-conjugated mitochondrial transcription factor-A (TFAM, Fig. 2D, E, F). The RoGFP-stained tubules of mitochondrial reticulum are only partly covered by the ND5 probe signal. In turn, the EGFP-TFAM-stained nucleoids of mitochondrial DNA (mt nucleoids) appear as numerous small sphere projections. In certain samples, the ND5 probe signal was apparently more intense (concentrated) in those $\mathrm{mt}$ nucleoid spots (Fig. 2D). The EGFP-TFAM fluorescence also co-localized with the 7S DNA probe emission (Fig. $2 \mathrm{G}, \mathrm{H}, \mathrm{I})$ and with the proximal D-loop probe emission

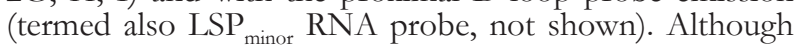
hardly distinguishable from the background, the signal of these two D-loop probes was less spread within the region of apparent mitochondrial reticulum tubules, i.e., the matrix space.

\section{Distinction between the three molecular beacon probes}

A comparison of Fig. 2D vs. 2G illustrates the difference between the ND5 and 7S DNA probes. Besides $\mathrm{mt}$ nucleoids, the ND5 probe hybridizes against the vast amount of ND5 mRNA (and polycistronic HSP RNA detached from the site of transcription, i.e., from $\mathrm{mt}$ nucleoids) spread inside the tubules of mitochondrial reticulum. Unlike for the ND5 probe, a more punctate character of images was observed for the 7S DNA probe and the proximal D-loop probe (termed also the $\mathrm{LSP}_{\text {minor }}$ RNA probe; Fig. 3E, 3I, vs. 3A). The numerous small spots most probably represent mt nucleoids as suggested by their overlay with EGFP-TFAM (Fig. $2 \mathrm{G}, \mathrm{H}, \mathrm{I})$. The stronger $\mathrm{mt}$ nucleoid signal relative to the overall mitochondrial (matrix space) tubule staining

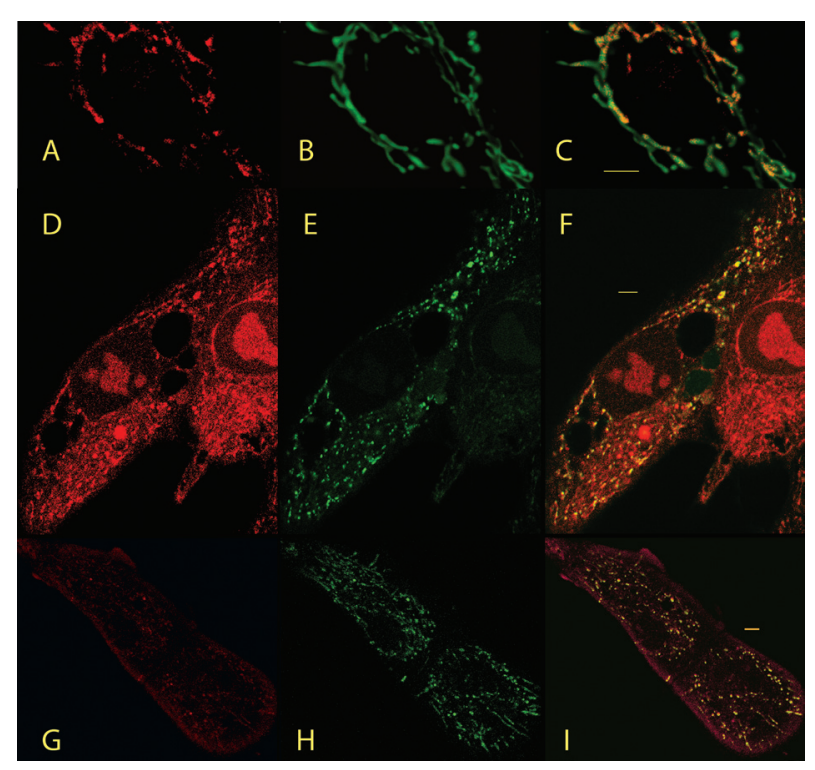

Figure 2. Colocalization of molecular beacon probes with tubules of mitochondrial reticulum and with mtDNA nucleoids. Confocal images of (A) ND5 molecular beacon probe; (B) matrixaddressed RoGFP; (C) overlay of A plus B illustrate co-localization of the ND5 probe with tubules of mitochondrial reticulum. Confocal images of (D) ND5 probe and (G) 7S DNA probe show more punctate character of the 7S DNA probe image and its similarity to the EGFP-TFAM-visualized $\mathrm{mt}$ nucleoids ( $\mathbf{E}$ and $\mathbf{H}$ ). Overlays show clearly that the ND5 probe hybridizes also to ND5 mRNA spread (plus to HSP RNA released from mt nucleoids) all over the matrix of mitochondrial reticulum tubules $(\mathbf{F})$, whereas the $7 \mathrm{~S}$ DNA probe is more concentrated in mt nucleoids (I). Scale bars at images represent $5 \mu \mathrm{m}$. The red pseudo color is used for molecular beacon probes.

observed for both D-loop probes would be compatible with a stronger degradation of LSP RNA in comparison with that of ND5 mRNA (or else HSP RNA).

\section{Transcribed polycistronic RNAs as possibly resolved structures}

Despite the only about $250 \mathrm{~nm}$ resolution of conventional confocal microscopy, distinct images were gathered with the three molecular beacon probes in combination with post-fixation (but pre-hybridization) treatment with RNases $\mathrm{A}$ and $\mathrm{H}$ and DNase I. In order to ascertain these differences, we performed the following three digestion treatments:

i) with RNase A, digesting ssRNA, which converted the ND5 probe images covering nearly whole mitochondrial tubules (Fig. 3A) into a punctate pattern observed in the $\mathrm{mt}$ nucleoid images (Fig. 3B). The pre-hybridization treatment with RNase A affected images obtained with the 7S DNA probe (Fig. 3E vs. F) and with the proximal D-loop probe (termed also $\mathrm{LSP}_{\text {minor }}$ RNA; Fig. 3I vs. J) to a lesser extent. This confirm that these two probes mainly visualize $\mathrm{mt}$ nucleoids.

ii) The treatment with RNase A plus RNase $H$ (the latter digesting RNA from RNA/DNA hybrids) led in general to a partial reversal of the punctate effect, giving a more diffuse signal of all three probes in comparison with that obtained after treatment with RNase A alone (Fig. 3B vs. C; 3F vs. G; J vs. K). We speculate that partially digested RNA structures spread in the mitochondrial tubules (in spite of the sample being fixed). Partial resistance to RNase A could be a feature of structures such as polycistronic LSP RNA (in the case of D-loop probes). Since 


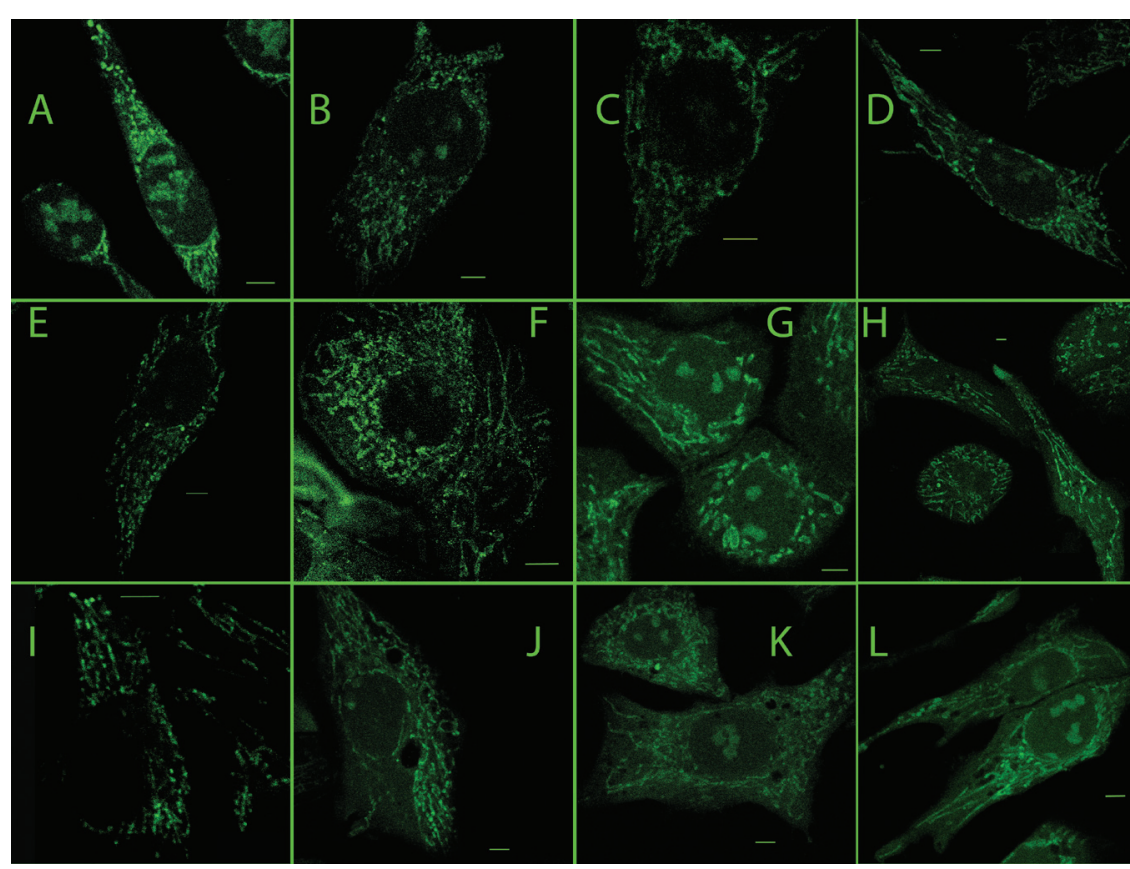

Figure 3. Confocal images of beacon probes before and after treatment with RNase A, RNase A plus RNase $\mathrm{H}$, or DNase I.

Confocal images are shown for ND5 probe (A) to (D); 7S DNA probe (E) to (H); and proximal D-loop probe (annealing also to LSP RNA) (I) to (L) ) in the absence of any further treatment (A), (E), (I); after post-fixation pre-hybridization treatment with RNase A, digesting ssRNA (B), (F), (J); after treatment with RNase A plus RNase $\mathrm{H}$, the latter digesting RNA from RNADNA hybrids (C), (G), (K); and after treatment with DNase I plus RNase H (D), (H), (L). Scale bars at each image represent $5 \mu \mathrm{m}$. Note that unlike in Fig. 2, now the green channel is used for molecular beacon probes.

ND5 mRNA could be digested by RNase A alone, only HSP RNA might be released by the additional action of RNase $\mathrm{H}$ in the case of the ND5 probe, and thus the effect was less pronounced in this case (Fig. 3C).

Finally, iii) treatment with DNase I plus RNase $\mathrm{H}$ without (Fig. 3D, H, L) or with a preceding RNase A treatment (not shown) converted images of mt nucleoids to the images of the whole mitochondrial network reticulum for all three probes. It reflects the distribution of the partially digested RNA and DNA all over the mitochondrial tubules in these fixed samples.

\section{DISCUSSION}

We have developed an in situ hybridization procedure for mitochondrial nucleic acids (mtFISH) with molecular beacon probes and verified it using conventional confocal microscopy. The anti L strand ND5 DNA\&RNA probe most likely anneal to the L-strand of ds mtDNA, thus highlighting the $\mathrm{mt}$ nucleoids, plus to the singlegene (ND5) mRNA spread all over the matrix space, i.e., within the tubules of mitochondrial reticulum. A minor contribution to a similar pattern may arise from hybridization with polycistronic HSP-RNA (that might be still attached to nucleoids and may be present in RITOLS, but mostly is spread into the entire matrix space). Distinct spots were present in the images visualized by the ND5 probe, which were co-localized with $\mathrm{mt}$ nucleoids (Holt et al., 2007; Bogenhagen et al., 2008). Moreover, the diffuse signal, most probably originating from the annealing to HSP-RNA and ND5 messenger RNA, disappeared after the RNase A pre-hybridization diges- tion of ssRNAs. Some signal was also observed in nuclei, most likely due to the presence of multiple insertions of mitochondrial genes into the nuclear chromosomes in nontranscribed regions.

Both D-loop probes used behaved in accordance with the simple assumption that the corresponding polycistronic and cleaved LSP RNA (the cleavage occurs due to self-processing at tRNA gene sites upon transcription) is degraded more extensively than the polycistronic and cleaved HSP RNA (tRNAs plus messenger RNAs for all mitochondrial protein coding genes but ND6). Indeed, both D-loop probes exhibited images of a more punctate character, showing numerous spots resembling $m t$ nucleoids. The EGFPTFAM-visualized $\mathrm{mt}$ nucleoids colocalized with the majority of signal of both D-loop probes. We expected that the $7 \mathrm{~S}$ DNA probe, which could anneal to three nucleic acid entities (Fig. $1 \mathrm{~A}, \mathrm{~B})$, would provide less distinct $\mathrm{mt}$ nucleoid images than the proximal D-loop probe (annealing also to the correspond-

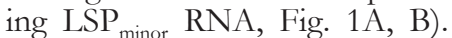
This assumption can only be verified after adaptation of the molecular beacon technique to super-resolution microscopy.

We also combined our mtFISH protocol with the post-fixation and pre-hybridization treatment with RNase A, RNase H, DNase I, and their combinations. A "contrasting" action of RNase A was observed for all probes, but it was most pronounced for the ND5 probe. The RNase simply eliminated messenger RNAs and other RNAs spread in the matrix space of mitochondrial tubules within the fixed sample. We speculate that some $\mathrm{mt}$ nucleoids exhibiting a higher signal might be replicating (but the presence of replication machinery had to be proven in them - such a search is out of scope of the current work). The "contrasting" action of RNase $\mathrm{H}$, however, indicates the presence of polycistronic LSP RNA (in the case of two D-loop probes) and HSP RNA (in the case of the ND5 DNA\&RNA probe) attached to $\mathrm{mt}$ nucleoids. We expect that $\mathrm{RNase} \mathrm{H}$ would digest presumed "transcription clouds" (not to be resolved in principle by the conventional confocal microscopy) around the $\mathrm{mt}$ nucleoids, representing those attached RNAs. The distinction of these transcriptional states (as well as RITOLS and replicating nucleoids) remains a future challenge for super-resolution microscopy in combination with analogs of our developed probes. Indeed, perspectives for the use of the developed probes lie in their use in conjunction with super-resolution microscopy such as stimulated emission depletion (STED) microscopy (Hell, 2007), for which modified probes (conjugated with STED-active dyes) may help to better resolve intra-nucleoid structure and might even detect RITOLS and R-loops, which are, unlike regular transcripts, pres- 
ent in single copies. This would broaden our knowledge of these structures and their relations to the resting state, transcribing state, and replicating state of $\mathrm{mt}$ nucleoids. In conclusion, the two developed D-loop molecular beacon probes represent a novel tool for mt nucleoid visualization.

\section{Acknowledgements}

Dr. Gabrielle Saretzki (Newcastle University, UK), for introducing us to molecular beacon system, and Dr. Werner Koopman (Nijmegen Centre for Molecular Life Sciences, The Netherlands), for providing baculoviral particles coding for EGFP-TFAM, are gratefully acknowledged, as well as cell culturing by Lenka Josková.

Financial support: This work was supported by grants No. P302/10/0346 (to P.J.) and P304/10/P204 (to A.D.) from the Grant Agency of the Czech Republic; and grants No. KJB500110902 (to J.J.) and AV0Z50110509 (to Inst. Physiology) from the Academy of Sciences of the Czech Republic.

\section{REFERENCES}

Andrews RM, Kubacka I, Chinnery PF, Lightowlers RN, Turnbull DM, Howell N (1999) Reanalysis and revision of the Cambridge reference sequence for human mitochondrial DNA. Nat Genet 23: 147.

Bogenhagen DF, Rousseau D, Burke S (2008) The layered structure of human mitochondrial DNA nucleoids. I Biol Chem 283: 3665-3675.

Bowmaker M, Yang MY, Yasukawa T, Reves A, Jacobs HT, Huberman JA, Holt IJ (2003) Mammalian mitochondrial DNA replicates bidirectionally from an initiation zone. J Biol Chem 278: 50961-50969.

Brown TA, Clayton DA (2006) Genesis and wanderings: origins and migrations in asymmetrically replicating mitochondrial DNA. Cell Cycle 5: 917-921.

Brown TA, Cecconi C, Tkachuk AN, Bustamante C, Clayton DA (2005) Replication of mitochondrial DNA occurs by strand displacement with alternative light-strand origins, not via a strand-coupled mechanism. Genes Dev 19: 2466-2476.

Brown TA, Tkachuk AN, Clayton DA (2008) Native R-loops persist throughout the mouse mitochondrial DNA genome. $J$ Biol Chem 283: 36743-36751.

Chang DD, Clayton DA (1985) Priming of human mitochondrial DNA replication occurs at the light-strand promoter. Proc Natl Acad Sci USA 82: 351-355.

Chang DD, Hixson JE, Clayton DA (1986) Minor transcription initiation events indicate that both human mitochondrial promoters function bidirectionally. Mol Cell Biol 6: 294-301.

Clayton DA (2003) Mitochondrial DNA replication: what we know. IUBMB Life 55: 213-217.

Cree LM, Samuels DC, de Sousa Lopes SC, Rajasimha HK, Wonnapinij P, Mann JR, Dahl HH, Chinnery PF (2008) A reduction of mitochondrial DNA molecules during embryogenesis explains the rapid segregation of genotypes. Nat Genet 40: 249-254.

Falkenberg M, Larsson NG, Gustafsson CM (2007) DNA replication and transcription in mammalian mitochondria. Annu Rev Biochem 76: 679-699.

Fusté JM, Wanrooij S, Jemt E, Granycome CE, Cluett TJ, Shi Y, Atanassova N, Holt IJ, Gustafsson CM, Falkenberg M (2010) Mitochondrial RNA polymerase is needed for activation of the origin of light-strand DNA replication. Mol Cell 37: 67-78.

Hell SW (2007) Far-field optical nanoscopy. Science 316: 1153-1158.
Holt IJ (2009) Mitochondrial DNA replication and repair: all a flap. Trends Biochem Sci 34: 358-365.

Holt IJ (2010) Zen and the art of mitochondrial DNA maintenance. Trends Genet 26: 103-109.

Holt IJ, Lorimer HE, Jacobs HT (2000) Coupled leading- and laggingstrand synthesis of mammalian mitochondrial DNA. Cell 100: 515524.

Holt IJ, He J, Mao C-C, Boyd-Kirkup JD, Martinsson P, Sembongi H, Reyes A, Spelbrink JN (2007) Mammalian mitochondrial nucleoids: organizing an independently minded genome. Mitochondrion 7: 311-321.

Hyvärinen AK, Pohjoismäki JL, Reyes A, Wanrooij S, Yasukawa T, Karhunen PJ, Spelbrink JN, Holt IJ, Jacobs HT (2007) The mitochondrial transcription termination factor mTERF modulates replication pausing in human mitochondrial DNA. Nucleic Acids Res 35: 6458-6474.

Kajander OA, Karhunen PJ, Holt IJ, Jacobs HT (2001) Prominent mitochondrial DNA recombination intermediates in human heart muscle. EMBO Rep 2: 1007-1012.

Koopman WJH, Hink MA, Verkaart S, Visch HJ, Smeitink JAM, Willems PHGM (2007) Partial complex I inhibition decreases mitochondrial motility and increases matrix protein diffusion as revealed by fluorescence correlation spectroscopy. Biochim Biophys Acta 1767: 940-947.

Lu F, Selak M, O’Connor J, Croul S, Lorenzana C, Butunoi C, Kalman B. J. (2000) Oxidative damage to mitochondrial DNA and activity of mitochondrial enzymes in chronic active lesions of multiple sclerosis. Neurol Sci 177: 95-103.

Pohjoismäki JL, Holmes JB, Wood SR, Yang MY, Yasukawa T, Reyes A, Bailey LJ, Cluett TJ, Goffart S, Willcox S, Rigby RE, Jackson AP, Spelbrink JN, Griffith JD, Crouch RJ, Jacobs HT, Holt IJ (2010) Mammalian mitochondrial DNA replication intermediates are essentially duplex but contain extensive tracts of RNA/DNA hybrid. J Mol Biol 397: 1144-1155.

Reyes A, Yang MY, Bowmaker M, Holt IJ (2005) Bidirectional replication initiates at sites throughout the mitochondrial genome of birds. J Biol Chem 280: 3242-3250.

Rossignol R, Gilkerson R, Aggeler R, Yamagata K, Remington SI, Capaldi RA (2004) Energy substrate modulates mitochondrial structure and oxidative capacity in cancer cells. Cancer Res 64: 985-993.

Santangelo PJ, Nitin N, Bao G (2005) Direct visualization of mRNA colocalization with mitochondria in living cells using molecular beacons. I Biomed Opt 10: 044025-1-16.

Scaglia F, Wong L-JC (2008) Human mitochondrial transfer RNAs: Role of pathogenic mutation in disease. Muscle Nerve 37: 150-171.

Shadel GS, Clayton DA (1997) Mitochondrial DNA maintenance in vertebrates. Ann Rev Biochem 66: 409-435.

Stewart JB, Treter C, Elson JL, Wredenberg A, Cansu Z, Trifunovic A, Larsson N-G (2008) Strong purifying selection in transmission of mammalian mitochondrial DNA. PLoS Biology 6: e10.

Tuppen HA, Blakely EL, Turnbull DM, Taylor RW (2010) Mitochondrial DNA mutations and human disease. Biochim Biophys Acta 1797: 113-128.

Xu B, Clayton DA (1996) RNA-DNA hybrid formation at the human mitochondrial heavy-strand origin ceases at replication start sites: an implication for RNA-DNA hybrids serving as primers. EMBO J 15: 3135-3143.

Yang MY, Bowmaker M, Reyes A, Vergani L, Angeli P, Gringeri E, Jacobs HT, Holt IJ (2002) Biased incorporation of ribonucleotides on the mitochondrial L-strand accounts for apparent strand-asymmetric DNA replication. Cell 111: 495-505.

Yasukawa T, Reyes A, Cluett TJ, Yang MY, Bowmaker M, Jacobs HT, Holt IJ (2006) Replication of vertebrate mitochondrial DNA entails transient ribonucleotide incorporation throughout the lagging strand. EMBO J 25: 5358-5371.

Zhadanov SI, Grechanina EY, Grechanina YB, Gusar VA, Fedoseeva NP, Lebon S, Munnich A, Schurr TG (2007) Fatal manifestation of a de novo ND5 mutation: Insights into the pathogenetic mechanisms of mtDNA ND5 gene defects. Mitochondrion 7: 260-266. 